\title{
Novel Power Control Scheme for Target Tracking in Radar Network with Passive Cooperation
}

\author{
Jing TAN ${ }^{1,2}$, Chenguang SHI ${ }^{* 1}$, Jianjiang $\mathrm{ZHOU}^{1}$ \\ ${ }^{1}$ Key Laboratory of Radar Imaging and Microwave Photonics, Ministry of Education, Nanjing University of Aeronautics \\ and Astronautics, No. 29, Yudao Street, Qinhuai District, Nanjing city, Jiangsu Province, Nanjing 210016, P. R. China \\ ${ }^{2}$ Dept. of Information Engineering, Nanhang Jincheng College, No. 88, Hangjin Avenue, Lukou Sub-district, Jiangning \\ District, Nanjing city, Jiangsu Province, Nanjing 211156, P. R. China \\ *scg_space@163.com
}

Submitted March 20, 2017 / Accepted October 26, 2017

\begin{abstract}
Distributed radar network systems (DRNS) have been shown to provide significant performance improvement. With the recent development, radar network has become an attractive platform for target tracking. In practice, the netted radars in DRNS are supposed to maximize their transmitting power to achieve better target tracking performance, which may be in contradiction with low probability of intercept (LPI). This paper investigates the problem of adaptive resource scheduling based on time difference of arrival (TDOA) cooperation for target tracking by DRNS consisting of a dedicated radar netting station and multiple netted radars. Firstly, the standard interacting multiple model (IMM) algorithm incorporating extended Kalman filter (EKF) is improved by modifying the Markov transition probability with current measurements. Then, a novel resource scheduling strategy based on TDOA cooperation is presented, in which the LPI performance for target tracking in DRNS is improved by optimizing the radar revisit interval and the transmitted power for a predefined target tracking accuracy. The comparison of the predictive error covariance matrix and the expected error covariance matrix is utilized to control the radar netting station under intermittent-working state with TDOA cooperation. Due to the lack of analytical closed-form expression for receiver operating characteristics (ROC), we utilize several popular information-theoretic criteria, namely, Bhattacharyya distance, Kullback-Leibler (KL) divergence, J-divergence, and mutual information (MI) as the metrics for target detection performance in target tracking process. The resulting optimization problems which are associated with different information-theoretic criteria are unified under a common framework. The nonlinear programming (NP) based genetic algorithm (GA) or else known as NPGA is employed to encounter with the highly nonconvex and nonlinear optimization problems in the framework. Numerical results demonstrate that the proposed algorithm not only has excellent target tracking accuracy, but also has better LPI performance comparing to other methods.
\end{abstract}

\section{Keywords}

Low probability of intercept (LPI), resource scheduling, distributed radar network systems (DRNS), target tracking, time difference of arrival (TDOA)

\section{Introduction}

\subsection{Background and Motivation}

Distributed radar network systems (DRNS), also known as spatial distributed multiple-input multiple-output (MIMO) radar systems [1], [2], have been attracting contentiously growing attention towards practical use due to their advantage of signal and spatial diversities. In addition to aforementioned, considerable research has been conducted relevant to the potential use of radar networks for achieving systems performance improvement correlated to target detection [3-7], target localization [8], [9], target tracking [10-13], waveform design [14-16], sensor selection [17], and information extraction.

The research on distributed radar network architectures has received increasing impetus in recent years, which has been extensively studied from various perspectives [3-44]. In [3], the authors introduced the concept of distributed MIMO radar and investigated the inherent performance limitations of both conventional phased array radars and the newly proposed radars. In [4], the problem of code design to improve the detection performance of multi-static radar in the presence of clutter was studied, where the information-theoretic criteria were used as design metrics. Niu et al. [8] developed the localization and tracking algorithms for noncoherent MIMO radar systems, in which it was demonstrated that the noncoherent MIMO radar can provide a significant performance improvement over traditional monostatic phased array radar with high range and azimuth resolutions. The work in [11] addressed 
the problem of sensor scheduling and power allocation in a cognitive radar network for multiple-target tracking (MTT). Reference [12] extended the system model in [11] and presented a performance-driven power allocation strategy for Doppler-only target tracking in unmodulated continuous wave (UCW) radar network, where the Bayesian Cramer-Rao lower bound was derived and utilized as an optimization criterion for the optimal power allocation scheme. In [14], the authors investigated the problem of target tracking in a multistatic radar system from the perspective of adaptive waveform selection, in which the transmitted waveform parameters were selected to minimize the target tracking covariance matrix. Overall, the previous studies lay a solid foundation for the problem of performance optimization in DRNS, and it should be pointed out that the target tracking performance improvement can be obtained with an increase of either the transmitted power or the number of radar nodes.

Today the notion of low probability of intercept (LPI) design is of high importance, which has become an essential part of military operations in modern electronic warfare In order to achieve LPI performance, it is necessary to dynamically manage the radar resources while guaranteeing a specified target tracking performance. Technically speaking, low transmit power, short dwell time, large revisit interval, and ultra-low side lobe antenna will lead to better LPI performance. Thus, the problem of LPI based radar resource scheduling in traditional monostatic radars has been extensively studied from various perspectives [20-26][26]. In [24], a novel radar energy control algorithm based on an improved interacting multiple model particle filtering (IMMPF) was formulated for LPI performance enhancement, which controlled the radiation time and power of radar according to the target's range and radar cross section (RCS) with a given probability of target detection. Later, Zhang et al. [25] developed an optimal sensor selection strategy based on passive sensor cooperation, and it was shown that the proposed algorithm can decrease the radar radiation times with excellent target tracking accuracy. Chen et al. extended the results of the previous research and presented a radar radiation control strategy for multiple aircraft platforms based on time difference of arrival (TDOA) cooperation [26], which utilized the comparison of covariance and the predefined threshold to control the radiation state [27]. Overall speaking, the reported works verified that adaptive transmit parameters control was an effective technique to improve the LPI performance for radar systems. However, the previous studies all focused on the monostatic radar. Applying this idea to the distributed radar networks will face a number of technical problems. Shi et al. addressed the LPI optimization strategies in radar networks for the first time [28-30], where it has been demonstrated that radar network architectures with multiple transmitters and receivers can provide remarkable LPI performance advantages over traditional monostatic radar system (MRS), and has triggered a resurgence of interest in DRNS. The authors in [31-33] investigated the sensor scheduling algorithm of selecting and assigning sensors dynamically for target tracking, which can obtain a good tradeoff between the target tracking accuracy and the LPI performance. Nevertheless, almost all of those works focus on the single parameter optimization. On the basis of the research mentioned above, the problem of adaptive transmitted power control for target tracking in DRNS based on passive sensors cooperation, which has not been considered, needs to be investigated.

This paper aims to investigate the problem of adaptive resource scheduling based on TDOA cooperation for target tracking by DRNS, where a distributed radar network with one dedicated radar netting station and multiple netted radar nodes is considered. Specifically, the criterion of the proposed LPI optimization strategy is to minimize the total transmitted power, which is achieved by optimizing the radar revisit interval (which is defined as the period for the next update after the measurement at present time) and the transmitted power with TDOA cooperation for a given target tracking accuracy.

\subsection{Major Contributions}

The major contributions of this work are listed as follows:

(1) Motivated by the target tracking algorithm utilized in [15], an improved interacting multiple model (IMM) algorithm incorporating extended Kalman filter (EKF) is employed for single target tracking, in which the Markov transition probability is updated based on the current measurements;

(2) The main contribution of this work lies in the proposed resource scheduling scheme with TDOA cooperation for DRNS. The work in [26] concentrates on the maximization of the revisit interval based on TDOA cooperation, whereas the transmit power control is ignored. On the other hand, in [28], only the minimization of total transmitted power for radar networks is considered. From the previous results in [26], [28], we can conclude that the revisit interval affects the target tracking accuracy, and the transmitted power affects the target detection performance during tracking process. Therefore, the LPI performance for target tracking in DRNS can be improved by jointly optimizing the radar revisit interval and the transmitted power for a predefined target tracking accuracy, where the fewer number of total radiation times and lower transmitted power leads to smaller transmission power consumption. In this paper, the predictive error covariance matrix is used to generate a so-called uncertainty ellipse which describes the spatial variance distribution of an efficient target's state estimate at each time index [12]. As such, the predictive error covariance matrix is compared with the expected error covariance to control the dedicated radar netting station under intermittent-working state with TDOA cooperation. To be specific, if the expected target tracking accuracy is satisfied, the DRNS work in passive mode, and all the radars locate and track the target with TDOA method. If 
the expected target tracking accuracy is not satisfied, the DRNS work in active mode, in which the dedicated radar netting station transmits radar signals and all the radar nodes in DRNS receive and process the echoes that are reflected from the target;

(3) Even though the closed-form expressions for the probabilities of detection and false alarm can be obtained, the analytical closed-form expression for receiver operating characteristics (ROC) is not tractable for distributed radar network system [15], [34], [35], we utilize several information-theoretic criteria including Bhattacharyya distance, Kullback-Leibler (KL) divergence, J-divergence, and mutual information (MI) as the metrics for target detection performance in target tracking process, which have previously been derived in reference [28]. In this paper, the resulting optimization problems associated with different information-theoretic criteria are cast under a unified framework, and the nonlinear programming (NP) based genetic algorithm (GA) or else known as NPGA is adopted to solve these problems, as the results in [28] suggest that NPGA can be extremely effective in solving the highly nonconvex and nonlinear optimization problem;

(4) Furthermore, we built a closed-loop tracker for target tracking in DRNS. We employ the improved IMMEKF algorithm to obtain an approximate estimate of the target state vector, where the initial target position and velocity can be obtained by passive sensors and known as prior information. Then, the radar network incorporates the predictive information in terms of target location and velocity into its task to form the radiation scheme, thereby establishing it a closed-loop system.

This paper is organized as follows. Section 2 describes the considered radar network system model and signal model. Section 3 presents the improved IMM-EKF algorithm. In Sec. 4, a novel adaptive resource scheduling algorithm for target tracking in DRNS based on TDOA cooperation is formulated, and the resulting highly nonconvex and nonlinear optimization problems associated with different information-theoretic criteria are considered under a unified framework and solved through the nonlinear programming based on genetic algorithm (NPGA). The numerical simulations are provided in Sec. 5. Finally, conclusion remarks are reported in Sec. 6.

\section{Modelling Architecture}

\subsection{System Model}

In this paper, we consider a distributed radar network consists of one dedicated radar netting station and $N_{\mathrm{r}}-1$ netted radars, which are located at different sites as depicted in Fig. 1 [1]. The DRNS can work in two modes, i.e., passive mode and active mode. When the DRNS work in passive mode, the dedicated radar netting station doesn't transmit any signals, and all the radar nodes in DRNS locate and track the target by receiving the signal $s(t)$ radiated from the target. Herein, it is supposed that the netted

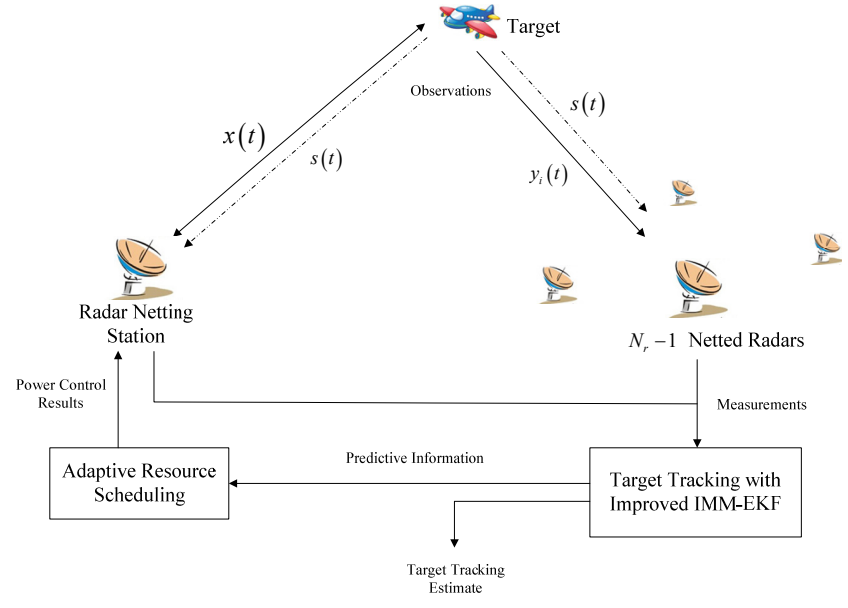

Fig. 1. Distributed radar network systems (Shi et al. in [37]).

radars in DRNS can receive the signals radiated from the target all the time, so that the passive TDOA method [36] can be employed to locate and track the target.

When the DRNS work in active mode, the dedicated radar netting station transmits radar signals and all the radars in DRNS can receive and process the echoes that are reflected from the target. The DRNS can be broken into $1 \times N_{\mathrm{r}}$ transmitter-receiver pairs each with a bistatic component contributing to the total signal-to-noise ratio (SNR) of the radar network.

We assume that the DRNS have a common precise knowledge of space and time. The total SNR in DRNS can be obtained by aggregating the SNR of each transmit-receive pair as follows [2]:

$$
S N R_{\mathrm{DRNS}}=\sum_{i=1}^{N_{\mathrm{r}}} \frac{P_{\mathrm{t}} G_{\mathrm{t}} G_{\mathrm{r} i} \sigma_{\mathrm{t} i} \lambda^{2}}{(4 \pi)^{3} k T_{\mathrm{o} i} B_{\mathrm{r}} F_{\mathrm{r} i} R_{\mathrm{t}}^{2} R_{\mathrm{r} i}^{2} L_{i}}
$$

where $P_{\mathrm{t}}$ is the transmitter power of the dedicated radar netting station, $G_{\mathrm{t}}$ is the transmit antenna gain, $G_{\mathrm{r} i}$ is the $i$ th receive antenna gain, $\sigma_{\mathrm{t} i}$ represents the radar cross section (RCS) of the target for the dedicated radar netting station and the $i$ th receiver, $\lambda$ represents the transmitted wavelength, $k$ and $T_{\mathrm{o} i}$ are Boltzmann's constant and the receiving system noise temperature at the $i$ th receiver respectively, $B_{\mathrm{r}}$ denotes the bandwidth of the matched filter for the transmitted waveform, $F_{\mathrm{r} i}$ denotes the noise factor for the $i$ th receiver, $L_{i}$ denotes the system loss between the dedicated radar netting station and the $i$ th receiver, $R_{\mathrm{t}}$ and $R_{\mathrm{r} i}$ are the distance from the dedicated radar netting station to the target and the distance from the target to the $i$ th receiver respectively.

\subsection{Signal Model}

Consider the $i$ th bistatic channel between the dedicated radar netting station and the $i$ th receiver. Based on the definitions in [44], [45], the path gain contains the target reflection coefficient $g_{i}$ and the propagation loss factor $p_{i}$. Let $g_{i}$ denote the target reflection gain between the dedicated radar netting station and the $i$ th receiver. It is 
assumed that the target is comprised of a large number of independent and identically distributed (i.i.d.) random scatterers (i.e. Swerling-I model and Swerling-II model). Based on the central limit theorem, we can model $g_{i}$ 's as i.i.d. zero-mean complex Gaussian random variables, that is, $g_{i} \sim C N\left(0, \sigma_{\mathrm{g}}^{2}\right)$, where $C N\left(\mu, \sigma^{2}\right)$ denotes a Gaussian distribution with mean $\mu$ and variance $\sigma^{2}$. The propagation loss factor $p_{i}$ is a function of antenna gain and waveform propagation distance, which can be expressed as [28], [44], [45]:

$$
p_{i}=\frac{\sqrt{G_{\mathrm{t}} G_{\mathrm{r} i}}}{R_{\mathrm{t}} R_{\mathrm{r} i}} .
$$

In this paper, when the DRNS work in active mode, we suppose that the transmitted waveform of the dedicated radar netting station is $\sqrt{P_{\mathrm{t}}} x(t)$, and then the received signal at the $i$ th receiver from a single target can be written as:

$$
y_{i}(t)=p_{i} g_{i} \sqrt{P_{\mathrm{t}}} x\left(t-\tau_{i}\right)+n_{i}(t)
$$

where $\int|x(t)|^{2} \mathrm{~d} t=1, \tau_{i}$ represents the time delay, $n_{i}(t)$ denotes the noise at receiver $i$. Without loss of generality and to simplify the analysis, it is assumed that the target is static over the observation period, thus no Doppler effect is considered. At the $i$ th receiver, the received signal is matched filtered by time response $x^{*}(-t)$, and the output signal can be expressed as [42]:

$$
\begin{aligned}
\tilde{y}_{i}(t) & =\int y_{i}(t) \cdot x^{*}(\tau-t) \mathrm{d} \tau \\
& =p_{i} g_{i} \sqrt{P_{\mathrm{t}}} \int x\left(\tau-\tau_{i}\right) \cdot x^{*}(\tau-t) \mathrm{d} \tau+\tilde{n}_{i}(t)
\end{aligned}
$$

where $\tilde{n}_{i}(t)=\int n_{i}(\tau) \cdot x^{*}(\tau-t) \mathrm{d} \tau$.

The discrete-time signal for the $i$ th receiver can be described as:

$$
r_{i} \triangleq \tilde{y}_{i}\left(\tau_{i}\right)=p_{i} g_{i} \sqrt{P_{\mathrm{t}}}+\theta_{i}
$$

where $r_{i}$ is the output of the matched filter at the $i$ th receiver sampled at $\tau_{i}, \theta_{i}=\tilde{n}_{i}\left(\tau_{i}\right)$, and $\theta_{i} \sim C N\left(0, \sigma_{\theta}^{2}\right)$.

With all the received signals, the target detection for DRNS leads to a binary hypothesis testing problem, with $H_{1}$ corresponding to the target presence hypothesis and $H_{0}$ corresponding to the null hypothesis:

$$
\left\{\begin{array}{l}
H_{0}: r_{i}=\theta_{i} \\
H_{1}: r_{i}=p_{i} g_{i} \sqrt{P_{\mathrm{t}}}+\theta_{i}
\end{array}\right.
$$

where $1 \leq i \leq N_{\mathrm{r}}$. The target detection problem can be formulated and solved by comparing the likelihood ratio test (LRT) function as follows [45]:

$$
\left\{\begin{array}{l}
H_{0}: T \triangleq \prod_{i=1}^{N_{\mathrm{r}}} \frac{f\left(r_{i} \mid H_{1}\right)}{f\left(r_{i} \mid H_{0}\right)}<\delta \\
H_{1}: T \triangleq \prod_{i=1}^{N_{\mathrm{r}}} \frac{f\left(r_{i} \mid H_{1}\right)}{f\left(r_{i} \mid H_{0}\right)}>\delta
\end{array}\right.
$$

where $\delta$ denotes a certain detection threshold for the hypothesis testing for the radar receivers.

\section{Improved IMM-EKF Algorithm}

\subsection{Target Dynamic Model}

Consider a two-dimensional target tracking scenario. The target state is given by $\mathbf{X}(k)=[x(k), \dot{x}(k), y(k), \dot{y}(k)]^{\mathrm{T}}$, where $[x(k), y(k)]$ denotes the target position, $[\dot{x}(k), \dot{y}(k)]$ denotes the target velocity, and the superscript $\mathrm{T}$ represents matrix transpose. The target dynamic model can be described as:

$$
\mathbf{X}(k)=\mathbf{F X}(k-1)+\mathbf{N}(k-1)
$$

where $\mathbf{F}$ is the transition matrix. The term $\mathbf{N}(k-1)$ in (8) represents the process noise of target motion, and is supposed to be an additive Gaussian noise vector with a covariance $\mathbf{Q}(k-1)=\mathrm{E}\left[\mathbf{N}(k-1) \mathbf{N}(k-1)^{\mathrm{T}}\right][15]$, where $\mathrm{E}[\bullet]$ denotes the mathematical expectation.

\subsection{Estimation Model}

For radar application, the target estimation model usually consists of target's distance and azimuth angle, which are thus nonlinear. Then, the nonlinear estimation equation can be given by:

$$
\mathbf{Z}(k)=\mathbf{h}(k, \mathbf{X}(k))+\mathbf{W}(k)
$$

where $\mathbf{Z}(k)$ is the estimation vector at time $k, \mathbf{W}(k)$ stands for the estimation error, and $\mathbf{h}(\bullet)$ is a vector of nonlinear transformation function.

It is known to all that different mode has different observation vector. In this paper, one dedicated radar netting station and $\left(N_{\mathrm{r}}-1\right)$ netted radar nodes are considered for single target tracking, where the passive mode can only provide the angle measurements including the azimuth angle and the elevation angle, while the active mode can provide both range and angle measurements for target tracking. Herein, for the sake of simplicity, we consider a two-dimensional target tracking scenario, where the target's elevation angle is assumed to be zero. In our case, $N_{\mathrm{r}}$ netted radars are employed to track a single target with TDOA method [36]. In order to locate a $2 \mathrm{D}$ target, the TDOA system should comprise three stations at least. The measurements received from two slave stations must be sent to the master station, where the time difference and the target position estimate are computed.

It is assumed that the positions of the master and slave stations are $\left(x_{0}, y_{0}\right)$ and $\left(x_{i}, y_{i}\right), i=1,2$, respectively. The TDOA equations can be given by:

$$
\left\{\begin{array}{l}
r_{i}^{2}(k)=\left[x(k)-x_{i}\right]^{2}+\left[y(k)-y_{i}\right]^{2} \\
r_{0}^{2}(k)=\left[x(k)-x_{0}\right]^{2}+\left[y(k)-y_{0}\right]^{2}
\end{array}\right.
$$


where $r_{0}(k)$ denotes the range between the target and the master station, $r_{i}(k)$ denotes the range between the target and the $i$ th slave station. Then, we can have that:

$$
\Delta r_{i}(k)=r_{i}(k)-r_{0}(k)=c \Delta t_{i 0}(k)
$$

where $c$ represents the speed of electromagnetic transmission. $\Delta t_{i 0}(k)$ stands for the corresponding time difference of $\Delta r_{i}(k)$ :

$$
\left\{\begin{array}{l}
\Delta t_{10}(k)=t_{1}(k)-t_{0}(k) \\
\Delta t_{20}(k)=t_{2}(k)-t_{0}(k)
\end{array}\right.
$$

where $t_{0}(k)$ and $t_{i}(k), \mathrm{i}=1,2$, are the time when the target's radiated signal is received by the master and slave stations, respectively. The observation vector of the passive mode can be described as:

$$
\mathbf{Z}_{\mathrm{PM}}(k)=\left[\begin{array}{c}
\Delta r_{1}(k) \\
\Delta r_{2}(k)
\end{array}\right]=c\left[\begin{array}{c}
t_{1}(k)-t_{0}(k) \\
t_{2}(k)-t_{0}(k)
\end{array}\right]+\mathbf{W}_{\mathrm{PM}}(k)
$$

where $\mathbf{W}_{\mathrm{PM}}(k)$ is the observation error with covariance $\mathbf{N}_{\mathrm{PM}}(k)$, and $\mathbf{h}_{\mathrm{PM}}(\bullet)$ represents the nonlinear transformation from the target state vector of target position in Cartesian coordinates to the observation vector of time difference.

Moreover, the observation vector of the active mode includes range measurement $r_{\mathrm{AM}}(k)$ and angle measurement $\theta_{\mathrm{AM}}(k)$, which can be written as:

$$
\mathbf{Z}_{\mathrm{AM}}(k)=\left[\begin{array}{c}
r_{\mathrm{AM}}(k) \\
\theta_{\mathrm{AM}}(k)
\end{array}\right]=\left[\begin{array}{c}
\sqrt{x^{2}(k)+y^{2}(k)} \\
\arctan \left[\frac{y(k)}{x(k)}\right]
\end{array}\right]+\mathbf{W}_{\mathrm{AM}}(k)(14)
$$

where $\mathbf{W}_{\mathrm{AM}}(k)$ represents the observation error with covariance $\mathbf{N}_{\mathrm{AM}}(k)$, and $\mathbf{h}_{\mathrm{AM}}(\bullet)$ is the nonlinear transformation from the target state vector of target position in Cartesian coordinates to the observation vector of range and azimuth angle.

\subsection{IMM-EKF Algorithm}

\subsubsection{Extended Kalman Filter}

The Kalman filter $(\mathrm{KF})$ is widely used in target tracking [38], [39]. However, the EKF and the unscented Kalman filter (UKF) [40] are more commonly employed, which is due to the fact that they can deal better with the nonlinearity between the measurement model and the target dynamic model, though they require much more expensive computational costs. The UKF has been demonstrated that it can provide the best tracking performance, but its computational cost is the highest.

Therefore, the standard EKF is employed for single target tracking problem in this paper because it can achieve the best tradeoff between its tracking performance and its computational cost [15]. The equations of EKF are provided as follows [15]:
Prediction:

$$
\begin{gathered}
\widehat{\mathbf{X}}(k \mid k-1)=\mathbf{F} \widehat{\mathbf{X}}(k-1 \mid k-1), \\
\mathbf{P}(k \mid k-1)=\mathbf{F P}(k-1 \mid k-1) \mathbf{F}^{\mathrm{T}}+\mathbf{Q}(k), \\
\mathbf{S}(k)=\mathbf{h}_{X}(k) \mathbf{P}(k \mid k-1) \mathbf{h}_{X}^{\mathrm{T}}(k)+\mathbf{R}(k) .
\end{gathered}
$$

Update:

$$
\begin{gathered}
\mathbf{K}(k)=\mathbf{P}(k \mid k-1) \mathbf{h}_{X}^{\mathrm{T}}(k) \mathbf{S}^{-1}(k), \\
\mathbf{P}(k \mid k)=\left[\mathbf{I}-\mathbf{K}(k) \mathbf{h}_{X}(k)\right] \mathbf{P}(k \mid k-1), \\
\widetilde{\mathbf{Z}}(k)=\mathbf{Z}(k)-\mathbf{h}(k, \widehat{\mathbf{X}}(k \mid k-1)), \\
\widehat{\mathbf{X}}(k \mid k)=\widehat{\mathbf{X}}(k \mid k-1)+\mathbf{K}(k) \widetilde{\mathbf{Z}}(k),
\end{gathered}
$$

where $\mathbf{h}_{X}(k)$ is a Jacobian of $\mathbf{h}(\mathbf{X}(k))$ evaluated at $\mathbf{X}=\widehat{\mathbf{X}}(k \mid k-1)$ [26]:

$$
\mathbf{h}_{X}(k)=\left[\nabla_{X} \mathbf{h}(k, \mathbf{X}(k))^{T}\right]_{\mathbf{X}=\hat{\mathbf{x}}(k \mid k-1)}^{\mathrm{T}} .
$$

To be specific, when the DRNS work in active mode,

$$
\begin{aligned}
\mathbf{h}_{X}^{\mathrm{AM}}(k) & =\left[\nabla_{X} \mathbf{h}(k, \mathbf{X}(k))^{\mathrm{T}}\right]_{\mathbf{X}=\hat{\mathbf{x}}(k \mid k-1)}^{\mathrm{T}} \\
& =\left[\begin{array}{cccc}
\frac{\hat{x}(k \mid k-1)}{\hat{r}} & 0 & \frac{\hat{y}(k \mid k-1)}{\hat{r}} & 0 \\
-\frac{\hat{y}(k \mid k-1)}{\hat{r}} & 0 & \frac{\hat{x}(k \mid k-1)}{\hat{r}} & 0
\end{array}\right]
\end{aligned}
$$

where

$$
\hat{r}=\sqrt{x^{2}(k \mid k-1)+y^{2}(k \mid k-1)} .
$$

While when the DRNS work in passive mode,

$$
\begin{aligned}
& \mathbf{h}_{X}^{\mathrm{PM}}(k)=\left[\nabla_{X} \mathbf{h}(k, \mathbf{X}(k))^{\mathrm{T}}\right]_{\mathbf{X}=\hat{\mathbf{x}}(k k-1)}^{\mathrm{T}}= \\
& {\left[\begin{array}{cccc}
\frac{\hat{x}(k \mid k-1)-x_{1}}{\hat{r}_{1}}-\frac{\hat{x}(k \mid k-1)-x_{0}}{\hat{r}_{0}} & 0 & \frac{\hat{y}(k \mid k-1)-y_{1}}{\hat{r}_{1}}-\frac{\hat{y}(k \mid k-1)-y_{0}}{\hat{r}_{0}} & 0 \\
\frac{\hat{x}(k \mid k-1)-x_{2}}{\hat{r}_{2}}-\frac{\hat{x}(k \mid k-1)-x_{0}}{\hat{r}_{0}} & 0 & \frac{\hat{y}(k \mid k-1)-y_{2}}{\hat{r}_{2}}-\frac{\hat{y}(k \mid k-1)-y_{0}}{\hat{r}_{0}} & 0
\end{array}\right]}
\end{aligned}
$$

where

$$
\left\{\begin{array}{l}
\hat{r_{0}}=\sqrt{\left[x(k \mid k-1)-x_{0}\right]^{2}+\left[y(k \mid k-1)-y_{0}\right]^{2}} \\
\hat{r_{1}}=\sqrt{\left[x(k \mid k-1)-x_{1}\right]^{2}+\left[y(k \mid k-1)-y_{1}\right]^{2}} \\
\hat{r_{2}}=\sqrt{\left[x(k \mid k-1)-x_{2}\right]^{2}+\left[y(k \mid k-1)-y_{2}\right]^{2}}
\end{array} .\right.
$$

\subsubsection{Interacting Multiple Model}

Herein, we employ the IMM method which incorporates three standard EKFs, where we consider three target dynamic models: 1) a constant velocity model, 2) a turn model with a positive turn rate, and 3) a turn model with a negative turn rate. The detailed descriptions of all the three dynamic models are given in Sec. 5 . 
The crucial feature of the resource management algorithm in radar network is that it must be predictive, where the predictive error covariance matrix enables us to make decisions in advance based on current knowledge. Given the predicted target state $\hat{\mathbf{X}}^{i}(k-1)$, model probability $u^{i}(k \mid k-1)$, and error covariance matrix $\mathbf{P}^{i}(k \mid k-1)$ at time index $k-1$, we can calculate the predictive error covariance matrix $\mathbf{P}_{\mathrm{pre}}^{\mathrm{IMM}}(k \mid k-1)$. Hence, the combined target state is given by:

$$
\widehat{\mathbf{X}}_{\mathrm{pre}}^{\mathrm{IMM}}(k \mid k-1)=\sum_{i=1}^{3} \widehat{\mathbf{X}}^{i}(k \mid k-1) u^{i}(k \mid k-1) .
$$

The predictive error covariance matrix can be computed as:

$$
\begin{aligned}
& \mathbf{P}_{\mathrm{pre}}^{\mathrm{IMM}}(k \mid k-1)= \\
& \sum_{i=1}^{3} u^{i}(k \mid k-1)\left\{\begin{array}{l}
\mathbf{P}^{i}(k \mid k-1) \\
+\left[\widehat{\mathbf{X}}^{i}(k \mid k-1)-\widehat{\mathbf{X}}_{\mathrm{pre}}^{\mathrm{IMM}}(k \mid k-1)\right] \\
{\left[\widehat{\mathbf{X}}^{i}(k \mid k-1)-\widehat{\mathbf{X}}_{\mathrm{pre}}^{\mathrm{IMM}}(k \mid k-1)\right]^{\mathrm{T}}}
\end{array}\right\} .
\end{aligned}
$$

It can be observed from (28) that there exists a restrictive relationship between the predictive error matrix and the revisit interval. To be specific, increasing the revisit interval leads to enlarging the trace of $\mathbf{P}_{\text {pre }}^{\mathrm{IMM}}(k \mid k-1)$, which results in the degradation of target tracking performance. Thus, a new measurement needs to be scheduled as soon as the tracking accuracy degrades to a specific level.

\subsection{Updated Markov Transmission Probabil- ity with Current Measurements}

In the previous standard IMM algorithm, the interacting process of inputs is a Markov process. The model transition governed by a first-order homogeneous Markov chain can be denoted as:

$$
P\left\{M(k)=m_{j} \mid M(k-1)=m_{i}\right\}=p_{i j}, i, j=1,2,3,
$$

where $p_{i j}$ is the Markov transition probability from model $m_{i}$ at time index $k-1$ to the model $m_{j}$ at time index $k$. In standard IMM algorithm, the interacting process is decided by the Markov transition probability matrix $\mathbf{P}_{\mathrm{t}}$, which is predetermined based on prior knowledge and remains the same in the target tracking process. However, because of target maneuver and distortion of the prior knowledge, the predefined Markov transition probability matrix cannot actually reflect the model transition of target motion, which results in target tracking error. For that reason, it is sufficient for us to utilize the current measurements to update the Markov transition probability matrix $\mathbf{P}_{\mathrm{t}}$.

Herein, it is supposed that the probability of model $j$ at time index $k-1$ is $u^{j}(k-1)$, and the probability of model $j$ at time index $k$ is $u^{j}(k)$. The difference of $u^{j}(k)$, and $u^{j}(k-1)$ characterizes the change of the degree of match with the target motion, which can be utilized to modify the Markov transition probability matrix $\mathbf{P}_{\mathrm{t}}$ [41], [43]. Thus, the change rate of model probability can be expressed as:

$$
\pi^{j}(k)=10^{\varpi^{j}(k)}, i=1,2,3,
$$

where

$$
\varpi^{j}(k)=\left[u^{j}(k)-u^{j}(k-1)\right]^{3} .
$$

It can be seen from (30) that when the probability of model $j$ increases, $\pi^{j}(k)>1$; otherwise, $\pi^{j}(k)<1$. We assume that the Markov transition probability at time index $k-1$ is $p_{i j}(k-1)$, then $\pi^{j}(k)$ is used to modify the Markov transition probability as follows:

$$
p_{i j}^{\prime}(k)=\pi^{j}(k) p_{i j}(k-1), i, j=1,2,3,
$$

which is different from the traditional EKF algorithm. It can be implied that the Markov transition probability can be increased with an increase in the parameter $\pi^{j}(k)$, which reflects the real-time target motion. Furthermore, the normalized Markov transition probability can be calculated as:

$$
p_{i j}(k)=\frac{p_{i j}^{\prime}(k)}{\sum_{j=1}^{3} p_{i j}^{\prime}(k)}=\frac{\pi^{j}(k) p_{i j}(k-1)}{\sum_{j=1}^{3} \pi^{j}(k) p_{i j}(k-1)}
$$

where

$$
\left\{\begin{array}{l}
0<p_{i j}<1 \\
\sum_{j=1}^{3} p_{i j}=1
\end{array}, i, j=1,2,3 .\right.
$$

According to (33), we know that the modified Markov transition probability is increased as $u^{j}(k)$ increases, which effectively avoids the problem of prior determination of the Markov transition probability matrix in standard IMM algorithm. The flow chart of the improved IMM-EKF algorithm with modified Markov transition probability is given in Fig. 2 [41], [43].

\section{Problem Formulation}

In this section, the LPI optimization strategy can be mathematically formulated as the problem of minimizing the total transmitted power subject to a predefined target tracking performance. With the improved IMM-EKF algorithm for target tracking, we are then in a position to design and minimize the total transmitted power in order to achieve effective LPI performance improvement in DRNS. It is introduced in [5] that the analytical closed-form expression for ROC does not exist. Thus, we used the information-theoretic criteria, namely, Bhattacharyya distance, KL divergence, J-divergence, and MI as the metrics for detection performance in target tracking process. In what follows, the corresponding LPI performance optimization based on TDOA cooperation associated with different information-theoretic criteria are cast under a unified frame- 


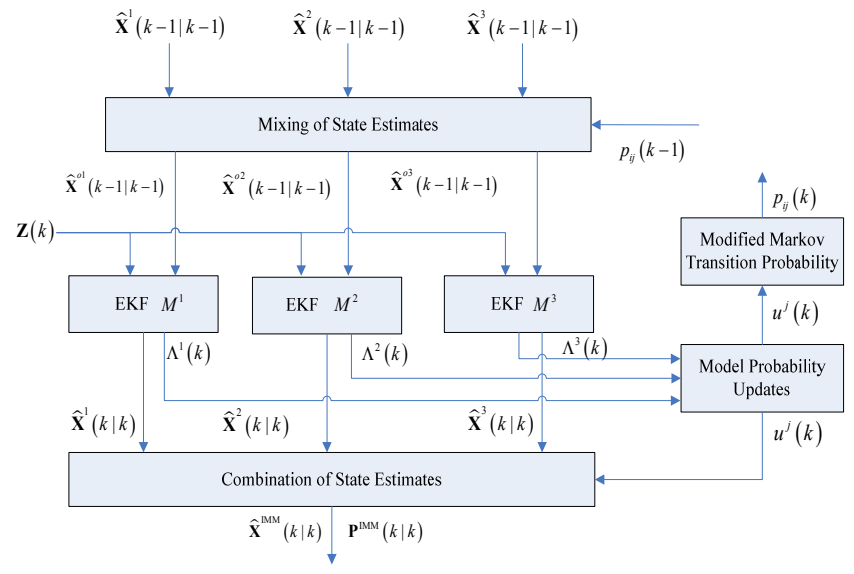

Fig. 2. Flow chart of the improved IMM-EKF algorithm (Guo et al. in [41]).

work and can be solved conveniently through NPGA. The general LPI optimization strategy is detailed as follows.

\subsection{Radiation Control}

In this paper, the improved IMM-EKF algorithm is adopted to track a single target. Actually, the term $\mathbf{P}_{\text {pre }}^{\text {IMM }}(k \mid k-1)$ in (28) implies the feedback information from the tracker to the DRNS, based on which the adaptive resource scheduling strategy can be implemented. Herein, we can utilize (28) to generate a so-called uncertainty ellipse, which describes the spatial variance distribution of an efficient target estimate [12].

Moreover, the expected covariance matrix is set to be $\lambda \cdot \mathbf{P}_{\mathrm{d}}(0<\lambda<+\infty)$, which denotes the lower bound on the error covariance of the estimation of the target position and velocity. The scalar $\lambda$ is defined as radar radiation control factor (RRCF), and it can be used to control target tracking accuracy.

Generally speaking, the uncertainty ellipse of $\mathbf{P}_{\text {pre }}^{\mathrm{IMM}}(k \mid k-1)$ would be contained in the uncertainty ellipse of $\lambda \cdot \mathbf{P}_{\mathrm{d}}$ if the expected target tracking accuracy is satisfied. Hence, it is sufficient for us to use $\left[\lambda \cdot \mathbf{P}_{\mathrm{d}}-\mathbf{P}_{\text {pre }}^{\mathrm{IMM}}(k \mid k-1)\right]$ as a criterion for the radar radiation control strategy. In other words, we can judge whether $\mathbf{P}_{\text {pre }}^{\mathrm{IMM}}(k \mid k-1)$ meets the requirement of $\lambda \cdot \mathbf{P}_{\mathrm{d}}$ according to whether $\left[\lambda \cdot \mathbf{P}_{\mathrm{d}}-\mathbf{P}_{\text {pre }}^{\text {IMM }}(k \mid k-1)\right]$ is positive semi-definite or not. The DNRS will work in active mode only when the expected target tracking accuracy is not satisfied, which means that the dedicated radar netting station will be used to radiate radar signals at time slot $k$ only when $\mathbf{P}_{\text {pre }}^{\mathrm{IMM}}(k \mid k-1)$ meets the following constraint:

$$
\lambda \cdot \mathbf{P}_{\mathrm{d}}-\mathbf{P}_{\mathrm{pre}}^{\mathrm{IMM}}(k \mid k-1)<\mathbf{0} .
$$

To be specific, if $\left[\lambda \cdot \mathbf{P}_{\mathrm{d}}-\mathbf{P}_{\text {pre }}^{\mathrm{IMM}}(k \mid k-1)\right] \geq \mathbf{0}$, the expected target tracking accuracy is satisfied. The DRNS work in passive mode and all the netted radars locate and track the target with TDOA method. While if $\left[\lambda \cdot \mathbf{P}_{\mathrm{d}}-\mathbf{P}_{\mathrm{pre}}^{\mathrm{IMM}}(k \mid k-1)\right]<\mathbf{0}$, the expected target tracking accuracy is not satisfied, and the DRNS work in active mode. The dedicated radar netting station transmits radar signals and all the radars in DRNS can receive and process the echoes that are reflected from the target. On the other hand, it is worth to point out that in the covariance control method, the uncertainty ellipse of the predictive error covariance matrix $\mathbf{P}_{\mathrm{pre}}^{\mathrm{IMM}}(k \mid k-1)$ may be not contained in the uncertainty ellipse of the expected error covariance matrix $\lambda \cdot \mathbf{P}_{\mathrm{d}}$ even though the trace of $\mathbf{P}_{\text {pre }}^{\mathrm{IMM}}(k \mid k-1)$ is smaller than that of $\lambda \cdot \mathbf{P}_{\mathrm{d}}$.

\subsection{Adaptive Transmit Power Control}

\subsubsection{Bhattacharyya Distance Criterion}

Bhattacharyya distance $B\left(p_{0}, p_{1}\right)$ measures the distance between two PDFs $p_{0}$ and $p_{1}$ [5]. It should be noted that the Bhattacharyya distance provides an upper bound on the probability of false alarm $p_{\mathrm{fa}}$ and at the same time yields a lower bound on the probability of detection $p_{\mathrm{d}}$.

Consider two multivariate Gaussian distributions $p_{0}$ and $p_{1}, p_{0} \sim C N\left(0, \Sigma_{0}\right)$ and $p_{1} \sim C N\left(0, \Sigma_{1}\right)$, the Bhattacharyya distance $B\left(p_{0}, p_{1}\right)$ can be expressed as:

$$
B\left(p_{0}, p_{1}\right)=\log \left\{\frac{\operatorname{det}\left[0.5\left(\Sigma_{0}+\Sigma_{1}\right)\right]}{\sqrt{\operatorname{det}\left(\Sigma_{0}\right) \operatorname{det}\left(\Sigma_{1}\right)}}\right\} .
$$

Let $B\left[f\left(\mathbf{r} \mid H_{0}\right), f\left(\mathbf{r} \mid H_{1}\right)\right]$ represent the Bhattacharyya distance between $H_{0}$ and $H_{1}$, where $f\left(\mathbf{r} \mid H_{0}\right)$ and $f\left(\mathbf{r} \mid H_{1}\right)$ are the PDFs of $\mathbf{r}$ under hypotheses $H_{0}$ and $H_{1}$, respectively. For the binary hypothesis testing problem, we can have that:

$$
\begin{aligned}
B_{\mathrm{DRNS}}(k) & \triangleq B\left[f\left(\mathbf{r} \mid H_{0}\right), f\left(\mathbf{r} \mid H_{1}\right)\right] \\
& =\sum_{i=1}^{N_{\mathrm{r}}} \log \left(\frac{1+0.5 \psi_{i}(k)}{\sqrt{1+\psi_{i}(k)}}\right) \\
& =\sum_{i=1}^{N_{\mathrm{r}}} \log \left[\frac{1+P_{\mathrm{t}}(k) R_{\mathrm{g}} p_{i}^{2}(k)\left(2 \sigma_{\theta}^{2}\right)^{-1}}{\sqrt{1+P_{\mathrm{t}}(k) \sigma_{\mathrm{g}}^{2} p_{i}^{2}(k)\left(\sigma_{\theta}^{2}\right)^{-1}}}\right]
\end{aligned}
$$

where $\psi_{i}(k)=P_{\mathrm{t}}(k) \sigma_{\mathrm{g}}^{2} p_{i}^{2}(k)\left(2 \sigma_{\theta}^{2}\right)^{-1}$.

It is discussed in [5] that maximization of the Bhattacharyya distance minimizes the upper bound on $p_{\text {fa }}$ while it maximizes the lower bound on $p_{\mathrm{d}}$. As expressed in (37), the Bhattacharyya distance derived here can be utilized to evaluate the target detection performance in DRNS as a function of different parameters, such as the transmitting power and the number of receivers in the system. The greater the Bhattacharyya distance between the two distributions of the binary hypothesis testing problem, the better the capability of DRNS to track the target [28], which would make the system more vulnerable in modern electronic warfare. 
Therefore, the Bhattacharyya distance can provide guidance to the problem of adaptive resource scheduling for target tracking in DRNS. According to (28) and (37), one can see that the target tracking accuracy is related to several parameters, including signal waveforms, revisited interval, and transmitted power. The adaptable parameters considered in this paper are the revisited interval and the transmitted power. For a predetermined target tracking performance at each time index, the aim of our work is to optimize the radar revisit interval and the transmitted power, which can result in the minimization of the total transmitted power, subject to the target tracking performance constraint. Eventually, the resulting LPI optimization problem can be formulated as follows:

$$
\left.\begin{array}{ll} 
& \min _{P_{\mathrm{t}}(k), N_{S}} \sum_{k=1}^{N_{\mathrm{s}}} P_{\mathrm{t}}(k), \\
\text { s.t. }: & B_{\mathrm{DRNS}}(k) \geq B_{\mathrm{th}}, \\
& \overline{P_{\min }} \leq P_{\mathrm{t}}(k) \leq \overline{P_{\max }}, \\
& \lambda \cdot \mathbf{P}_{\mathrm{d}}-\mathbf{P}_{\mathrm{pre}}^{\mathrm{IMM}}(k \mid k-1) \geq \mathbf{0}
\end{array}\right\}
$$

where $N_{\mathrm{S}}$ is the total times of radar radiation, $B_{\text {th }}$ is the Bhattacharyya distance threshold for target detection performance, and the transmitting power of the dedicated radar netting station at time index $k$ is constrained by a maximum value $\overline{P_{\max }}$ and a minimum value $\overline{P_{\min }}$.

\subsubsection{Kullback-Leibler Divergence Criterion}

The KL-divergence $D\left(p_{0} \| p_{1}\right)$ is another metric to measure the distance between two PDFs $p_{0}$ and $p_{1}$. Consider a binary hypothesis testing problem with $f\left(\mathbf{r} \mid H_{0}\right)$ and $f\left(\mathbf{r} \mid H_{1}\right)$. It is stated in the Stein Lemma that for any fixed value of $p_{\mathrm{fa}}$,

$$
D\left[f\left(\mathbf{r} \mid H_{0}\right) \| f\left(\mathbf{r} \mid H_{1}\right)\right]=\lim _{N \rightarrow \infty}\left[-\frac{1}{N} \log \left(1-p_{\mathrm{d}}\right)\right],
$$

which indicates that for any fixed $p_{\text {fa }}$, the maximization of the KL-divergence leads to asymptotic maximization of $p_{\mathrm{d}}$. From (39), we can observe that for any fixed $p_{\mathrm{fa}}$, the maximization of KL-divergence metric $D\left[f\left(\mathbf{r} \mid H_{0}\right) \| f\left(\mathbf{r} \mid H_{1}\right)\right]$ leads to an asymptotic maximization of $p_{\mathrm{d}}$. In addition, we can obtain that:

$$
D\left[f\left(\mathbf{r} \mid H_{0}\right) \| f\left(\mathbf{r} \mid H_{1}\right)\right]=-\mathrm{E}\left[\log (\Pi) \mid H_{0}\right]
$$

where $\Pi$ is the likelihood ratio defined as:

$$
\Pi \triangleq \frac{f\left(\mathbf{r} \mid H_{1}\right)}{f\left(\mathbf{r} \mid H_{0}\right)} .
$$

Using (39) and (40), the KL-divergence associated with (6) can be expressed as:

$$
\begin{aligned}
D_{\text {DRNS }}(k) & \triangleq D\left[f\left(\mathbf{r} \mid H_{0}\right) \| f\left(\mathbf{r} \mid H_{1}\right)\right] \\
& =\int f\left(\mathbf{r} \mid H_{0}\right) \cdot \log \frac{f\left(\mathbf{r} \mid H_{0}\right)}{f\left(\mathbf{r} \mid H_{1}\right)} \mathrm{d} \mathbf{r}
\end{aligned}
$$

$$
\begin{aligned}
D_{\text {DRNS }}(k) & \triangleq \sum_{i=1}^{N_{\uparrow}}\left\{\log \left[1+\psi_{i}(k)\right]-\frac{\psi_{i}(k)}{1+\psi_{i}(k)}\right\} \\
& =\sum_{i=1}^{N_{r}}\left\{\begin{array}{l}
\log \left[1+P_{\mathrm{t}}(k) \sigma_{\mathrm{g}}^{2} p_{i}^{2}(k)\left(\sigma_{\theta}^{2}\right)^{-1}\right] \\
-\frac{P_{\mathrm{t}}(k) \sigma_{\mathrm{g}}^{2} p_{i}^{2}(k)\left(\sigma_{\theta}^{2}\right)^{-1}}{1+P_{\mathrm{t}}(k) \sigma_{\mathrm{g}}^{2} p_{i}^{2}(k)\left(\sigma_{\theta}^{2}\right)^{-1}}
\end{array}\right\} .
\end{aligned}
$$

Similarly to problem (38), the KL-divergence based LPI optimization can be formulated as:

$$
\left.\begin{array}{ll} 
& \min _{P_{t}(k), N_{\mathrm{S}}} \sum_{k=1}^{N_{\mathrm{S}}} P_{\mathrm{t}}(k), \\
\text { s.t. : } & D_{\mathrm{DRNS}}(k) \geq D_{\mathrm{th}}, \\
& \overline{P_{\min }} \leq P_{\mathrm{t}}(k) \leq \overline{P_{\max }}, \\
& \lambda \cdot \mathbf{P}_{\mathrm{d}}-\mathbf{P}_{\mathrm{pre}}^{\mathrm{IMM}}(k \mid k-1) \geq \mathbf{0}
\end{array}\right\}
$$

where $D_{\text {th }}$ is the KL-divergence threshold for target detection performance.

\subsubsection{J-Divergence Criterion}

The J-divergence metric $J\left(p_{0}, p_{1}\right)$ is another measure of the distance between two PDFs $p_{0}$ and $p_{1}$. It is defined as follows:

$$
J\left(p_{0}, p_{1}\right) \triangleq D\left(p_{0} \| p_{1}\right)+D\left(p_{1} \| p_{0}\right) .
$$

The Stein Lemma introduces that in a binary hypothesis testing problem with with $f\left(\mathbf{r} \mid H_{0}\right)$ and $f\left(\mathbf{r} \mid H_{1}\right)$, and for any fixed $p_{\text {fa }}$, we can obtain:

$$
D\left[f\left(\mathbf{r} \mid H_{1}\right) \| f\left(\mathbf{r} \mid H_{0}\right)\right]=\lim _{N \rightarrow \infty}\left[-\frac{1}{N} \log \left(p_{\mathrm{fa}}\right)\right] .
$$

For the binary hypothesis testing problem in (6) with $f\left(\mathbf{r} \mid H_{0}\right)$ and $f\left(\mathbf{r} \mid H_{1}\right)$, we can write:

$$
\begin{aligned}
J & =\mathrm{E}\left\{(\Pi-1) \log (\Pi) \mid H_{0}\right\} \\
& =\left[\int \frac{f\left(\mathbf{r} \mid H_{1}\right)}{f\left(\mathbf{r} \mid H_{0}\right)} \log (\Pi) f\left(\mathbf{r} \mid H_{0}\right) \mathrm{d} \mathbf{r}\right]-\mathrm{E}\left[\log (\Pi) \mid H_{0}\right] \\
& =\mathrm{E}\left[\log (\Pi) \mid H_{1}\right]-\mathrm{E}\left[\log (\Pi) \mid H_{0}\right] .
\end{aligned}
$$

Using (46) along with the similar derivations as in the case of KL-divergence, the J-divergence associated with (6) can be given by:

$$
\begin{aligned}
J_{\text {DRNS }}(k) & \triangleq J\left[f\left(\mathbf{r} \mid H_{0}\right), f\left(\mathbf{r} \mid H_{1}\right)\right] \\
& =\sum_{i=1}^{N_{r}} \frac{\psi_{i}^{2}(k)}{1+\psi_{i}(k)} \\
& =\sum_{i=1}^{N_{r}} \frac{\left[P_{\mathrm{t}}(k) \sigma_{\mathrm{g}}^{2} p_{i}^{2}(k)\left(\sigma_{\theta}^{2}\right)^{-1}\right]^{2}}{1+P_{\mathrm{t}}(k) \sigma_{\mathrm{g}}^{2} p_{i}^{2}(k)\left(\sigma_{\theta}^{2}\right)^{-1}} .
\end{aligned}
$$

Consequently, the corresponding J-divergence based LPI optimization problem can be expressed as: 


$$
\left.\begin{array}{ll} 
& \min _{P_{t}(k), N_{S}} \sum_{k=1}^{N_{S}} P_{\mathrm{t}}(k), \\
\text { s.t. }: & J_{\mathrm{DRNS}}(k) \geq J_{\text {th }}, \\
& \overline{P_{\min }} \leq P_{t}(k) \leq \overline{P_{\max }}, \\
& \lambda \cdot \mathbf{P}_{\mathrm{d}}-\mathbf{P}_{\text {pre }}^{\mathrm{IMM}}(k \mid k-1) \geq \mathbf{0}
\end{array}\right\}
$$

where $J_{\text {th }}$ is the $\mathrm{J}$-divergence threshold for target detection performance.

\subsubsection{Mutual Information Criterion}

MI is another metric that has been widely used for radar transmit waveform design and optimization. The MI between the backscatter signal and the estimated target response statistically depends on the target features, which is often utilized as a design criterion [28]. It should be noted that the larger MI leads to target tracking performance improvements. The MI metric associated with (6) can be obtained as:

$$
\begin{aligned}
I_{\mathrm{DRNS}}(k) & \triangleq \sum_{i=1}^{N_{\mathrm{r}}} \log \left[1+\psi_{i}(k)\right] \\
& =\sum_{i=1}^{N_{\mathrm{r}}} \log \left[1+P_{\mathrm{t}}(k) \sigma_{\mathrm{g}}^{2} p_{i}^{2}(k)\left(\sigma_{\theta}^{2}\right)^{-1}\right] .
\end{aligned}
$$

Thus, the MI criterion based LPI optimization problem can be summarized as:

$$
\left.\begin{array}{ll} 
& \min _{P_{t}(k), N_{\mathrm{S}}} \sum_{k=1}^{N_{\mathrm{S}}} P_{\mathrm{t}}(k), \\
\text { s.t. }: & I_{\mathrm{DRNS}}(k) \geq I_{\mathrm{th}}, \\
& \overline{P_{\min }} \leq P_{t}(k) \leq \overline{P_{\max }}, \\
& \lambda \cdot \mathbf{P}_{\mathrm{d}}-\mathbf{P}_{\text {pre }}^{\mathrm{IMM}}(k \mid k-1) \geq \mathbf{0}
\end{array}\right\}
$$

where $I_{\text {th }}$ is the MI threshold for target detection performance.

\subsubsection{Unified Framework Based on NPGA}

Based on the above derivations, we can cast the various information-theoretic criteria based LPI optimization problems under a unified optimization framework. Thus, we formulate the following general form of the optimization problems in (38), (43), (48), and (50):

$$
\left.\begin{array}{ll} 
& \min _{P_{t}(k), N_{\mathrm{S}}} \sum_{k=1}^{N_{\mathrm{S}}} P_{\mathrm{t}}(k), \\
\text { s.t. }: & \Omega_{\mathrm{DRNS}}(k) \geq \Omega_{\mathrm{th}}, \\
& \overline{P_{\min }} \leq P_{t}(k) \leq \overline{P_{\max }}, \\
& \lambda \cdot \mathbf{P}_{\mathrm{d}}-\mathbf{P}_{\text {pre }}^{\mathrm{IMM}}(k \mid k-1) \geq \mathbf{0}
\end{array}\right\}
$$

where $\Omega_{\text {DRNS }} \in\left\{B_{\text {DRNS }}, D_{\text {DRNS }}, J_{\text {DRNS }}, I_{\text {DRNS }}\right\}$ and $\Omega_{\text {th }}$ is the corresponding threshold for target detection.

In this paper, the optimal solutions to the unified nonconvex, nonlinear, and constrained optimization problem
(51) can easily be obtained with NPGA, which has a good performance on the convergence speed, and it can improve the searching performance of ordinary genetic algorithm [28]. The NPGA is extremely effective in solving multidimensional optimization problems with non-convex and nonlinear constraints on the variables. Therefore, the optimal solutions can be calculated efficiently, such that the LPI techniques can be used in real-time systems. In Fig. 3, the flow chart of NPGA is provided, which can help us have an insight into this method.

Overall speaking, the closed-loop target tracking strategy in DRNS can be summarized as follows. Firstly, the target state at the current time index is obtained by the improved IMM-EKF algorithm. Then, the predictive error covariance matrix is calculated, based on which the radar radiation control scheme can be implemented. Finally, the adaptive transmitted power control result is sent back to guide the radiation scheme at the next time index [12],

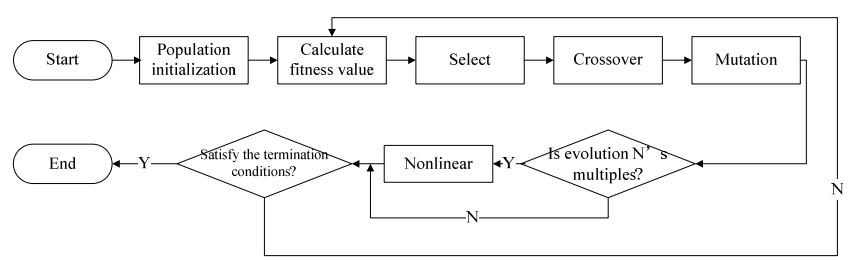

Fig. 3. Flow chart of NPGA procedure (Shi et al. in [28], [29]).

- Step 1: Initialization Phase: Given the target state estimate $\mathbf{X}(k-1)$ and the corresponding error covariance matrix $\mathbf{P}(k-1)$ at time $(k-1)$.

- Step 2: Prediction Phase: Determine the radar radiation time when the target tracking accuracy in the predicted state has degraded to the given level:

$$
\begin{aligned}
& \widehat{\mathbf{X}}_{\mathrm{pre}}^{\mathrm{IMM}}(k \mid k-1)=\sum_{i=1}^{3} \widehat{\mathbf{X}}^{i}(k \mid k-1) u^{i}(k \mid k-1), \\
& \mathbf{P}_{\mathrm{pre}}^{\mathrm{IMM}}(k \mid k-1)= \\
& \sum_{i=1}^{3} u^{i}(k \mid k-1)\left\{\begin{array}{l}
\mathbf{P}^{i}(k \mid k-1) \\
+\left[\hat{\mathbf{X}}^{i}(k \mid k-1)-\hat{\mathbf{X}}_{\mathrm{pre}}^{\mathrm{IMM}}(k \mid k-1)\right] \\
{\left[\hat{\mathbf{X}}^{i}(k \mid k-1)-\widehat{\mathbf{X}}_{\mathrm{pre}}^{\mathrm{IMM}}(k \mid k-1)\right]^{\mathrm{T}}}
\end{array}\right\} .
\end{aligned}
$$

- Step 3: LPI Optimization Phase: For DRNS, obtain the optimal transmitted power $P_{\mathrm{t}}(k)$ by solving the following LPI optimization problem:

$$
\left.\begin{array}{ll} 
& \min _{P_{t}(k), N_{\mathrm{S}}} \sum_{k=1}^{N_{\mathrm{S}}} P_{\mathrm{t}}(k), \\
\text { s.t. }: & \Omega_{\mathrm{DRNS}}(k) \geq \Omega_{\mathrm{th}}, \\
& \overline{P_{\min }} \leq P_{\mathrm{t}}(k) \leq \overline{P_{\max }}, \\
& \lambda \cdot \mathbf{P}_{\mathrm{d}}-\mathbf{P}_{\mathrm{pre}}^{\mathrm{IMM}}(k \mid k-1) \geq \mathbf{0}
\end{array}\right\}
$$

- Step 4: Measurement Phase: Make the measurement with the optimal parameters, update the target state $\hat{\mathbf{X}}^{\mathrm{IMM}}(k \mid k)$ and the corresponding error covariance matrix $\mathbf{P}^{\mathrm{IMM}}(k \mid k)$ by employing the improved IMM-EKF.

Tab. 1. General steps of the proposed algorithm. 


\begin{tabular}{|ll|}
\hline Radar & Locations \\
\hline Radar netting station & {$[0,0] \mathrm{km}$} \\
\hline Radar1 & {$[8,6] \mathrm{km}$} \\
\hline Radar2 & {$[-8,6] \mathrm{km}$} \\
\hline Radar3 & {$[0,10] \mathrm{km}$} \\
\hline
\end{tabular}

Tab. 2. Radar netting station and netted radars locations.

thereby rendering it a closed-loop system. The general steps of the proposed algorithm are provided in Tab. 1, which is a combination of various proposed techniques.

So far, we have completed the formulation of the TDOA cooperation based adaptive resource scheduling strategy for target tracking in DRNS. In what follows, some numerical simulations are dedicated to show the effectiveness of our presented method.

\section{Numerical Results}

In the sequel, numerical simulation results are dedicated to verify the accuracy of the theoretical derivations as well as to demonstrate the enhancement of the LPI performance brought by our proposed adaptive resource scheduling strategy. A distributed radar network with one dedicated radar netting station and three spatially distributed netted radars is considered. The locations of the dedicated radar netting station and netted radars are shown in Tab. 2.

Herein, we set the system parameters $G_{\mathrm{t}}=30 \mathrm{~dB}$, $G_{\mathrm{r} i}=30 \mathrm{~dB},\left(\forall i=1, \ldots, N_{\mathrm{r}}\right), \sigma_{\mathrm{g}}{ }^{2}=1$, and $\sigma_{\theta}{ }^{2}=10^{-10}$. The lower and upper bound of the dedicated radar netting station's transmitted power are set to be $\bar{P}_{\min }=0$ and $\bar{P}_{\max }=24 \mathrm{~kW}$. The threshold of target tracking performance $\Omega_{\text {th }}$ can be calculated in the condition that the dedicated radar netting station's transmitted power is $\bar{P}_{\max }=24 \mathrm{~kW}$ at a distance of $267 \mathrm{~km}$ between the dedicated radar netting station and the target, which is the marginal value of the basic performance requirement for target detection in target tracking process.

The improved IMM-EKF algorithm is employed in the simulation with three target dynamic models: 1) a constant velocity model $\left.F_{\mathrm{CV}}, 2\right)$ a coordinate turn model $F_{\mathrm{CT}}$ with positive turn rate $\omega=\pi / 180$, and 3 ) a coordinate turn model $F_{\mathrm{CT}}$ with negative turn rate $\omega=-\pi / 180$.

$$
\begin{gathered}
F_{\mathrm{CV}}=\left[\begin{array}{llll}
1 & T & 0 & 0 \\
0 & 1 & 0 & 0 \\
0 & 0 & 1 & T \\
0 & 0 & 0 & 1
\end{array}\right], \\
F_{\mathrm{CT}}=\left[\begin{array}{cccc}
1 & \frac{\sin (\omega T)}{\omega} & 0 & \frac{\cos (\omega T)-1}{\omega} \\
0 & \cos (\omega T) & 0 & -\sin (\omega T) \\
0 & \frac{1-\cos (\omega T)}{\omega} & 1 & \frac{\sin (\omega T)}{\omega} \\
0 & \sin (\omega T) & 0 & \cos (\omega T)
\end{array}\right],
\end{gathered}
$$

where $T$ is the time interval between successive frames. For all the models, the covariance matrix of the process noise is set to be:

$$
\mathbf{Q}(k-1)=\sigma^{2}\left[\begin{array}{cccc}
\frac{T^{4}}{4} & \frac{T^{3}}{2} & 0 & 0 \\
\frac{T^{3}}{2} & T^{2} & 0 & 0 \\
0 & 0 & \frac{T^{4}}{4} & \frac{T^{3}}{2} \\
0 & 0 & \frac{T^{3}}{2} & \frac{T^{4}}{2}
\end{array}\right]
$$

where $\sigma^{2}=0.04^{2}$. The covariance matrix of the measurement error:

$$
\mathbf{N}_{\mathrm{AM}}(k)=\left[\begin{array}{cc}
\sigma_{r}^{2} & 0 \\
0 & \sigma_{\beta}^{2}
\end{array}\right],
$$

$$
\mathbf{N}_{\mathrm{PM}}(k)=\sigma_{\mathrm{TDOA}}^{2}\left[\begin{array}{ccc}
1 & 0 & 0 \\
0 & 1 & 0 \\
0 & 0 & 1
\end{array}\right] \text {, }
$$

where $\sigma_{\mathrm{r}}=0.1 \mathrm{~km}, \sigma_{\beta}=0.001^{\circ}$, and $\sigma_{\mathrm{TDOA}}=3 \times 10^{-3} \mathrm{~km}$ [15].

The initial model probabilities are 0.95 for the target to be in the constant velocity model, 0.025 for the target to be in the coordinate turn model with positive turn rate, and 0.025 for the target to be in the coordinate turn model with negative turn rate. The initial model transition probability matrix is set to be:

$$
\mathbf{P}_{t}=\left[\begin{array}{lll}
0.98 & 0.01 & 0.01 \\
0.01 & 0.98 & 0.01 \\
0.01 & 0.01 & 0.98
\end{array}\right]
$$

The simulated target trajectory is illustrated in Fig. 4. The initial position and velocity of the simulated target are $[100,80] \mathrm{km}$ and $[0.4,0.3] \mathrm{km} / \mathrm{s}$, respectively. The target takes a right turn between $60 \mathrm{~s}$ and $100 \mathrm{~s}$, and takes a left turn between $140 \mathrm{~s}$ and $200 \mathrm{~s}$. The time interval between successive frames is $T=2 \mathrm{~s}$, and a sequence of 100 frames are utilized in the simulation.

To better show the optimality of our proposed strategy, Fig. 5 and Fig. 6 illustrate the root mean square error $(R M S E)$ and the average root mean square error (ARMSE) of the whole target tracking process, respectively. The $R M S E$ at the $k$ th tracking interval can be calculated as:

$$
\begin{aligned}
& \operatorname{RMSE}(k)= \\
& \sqrt{\frac{1}{N_{\mathrm{MC}}} \sum_{n=1}^{N_{\mathrm{MC}}}\left\{\left[x(k)-\hat{x}_{n}(k \mid k)\right]^{2}+\left[y(k)-\hat{y}_{n}(k \mid k)\right]^{2}\right\}}
\end{aligned}
$$

where $N_{\mathrm{MC}}$ is the number of Monte-Carlo trials, and $\left[\hat{x}_{n}(k \mid k), \hat{y}_{n}(k \mid k)\right]$ is the target state estimate at the $n$th trial. The ARMSE is defined as follows: 


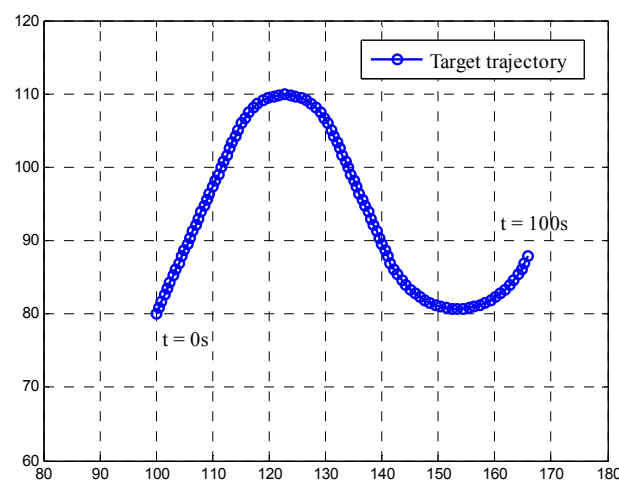

Fig. 4. Simulated target trajectory.

$$
A R M S E=\frac{1}{N_{\mathrm{T}}} \sum_{k=1}^{N_{\mathrm{T}}} R M S E(k)
$$

where $N_{\mathrm{T}}$ is the total frames in the simulation.

In Fig. 5 and Fig. 6, we compare the target tracking performance of the proposed algorithm and the covariance control algorithm averaged over 1000 Monte-Carlo trials. Besides, the proposed algorithm in [28] without TDOA cooperation is also included in this simulation, where the dedicated radar netting station is scheduled to radiate power in each time index. Obviously, the target tracking performance of the algorithm in [28] is the best. However, as shown later, the total times of radar radiation by employing the algorithm proposed in [28] is the largest, which in turn has the worst LPI performance. The proposed algorithm can obtain the best tradeoff between the target tracking accuracy and the total times of radar radiation. It can be seen from Fig. 5 that our proposed algorithm is strictly smaller than that of the covariance control algorithm.

Moreover, we can see from Fig. 5 and Fig. 6 that as the RRCF increases from $\lambda=1$ to $\lambda=3$ the target tracking performance degrades as expected. This is because that the expected error covariance matrix $\lambda \cdot \mathbf{P}_{\mathrm{d}}$ is increased with the increase of $\lambda$, which demonstrates that there exists a restrictive relationship between the RRCF and the target tracking accuracy.

Overall, the covariance control algorithm does not perform well compared to our proposed algorithm. This is due to the fact that the covariance control algorithm aims to minimize the trace of the predictive error covariance matrix, which is not a good representative metric for the target tracking accuracy.

In this simulation, we examine the radar real-time radiation state of the proposed algorithm with different RRCF. From Fig. 7, it can be observed that the total times of radar radiation is decreased as the RRCF increases, and the corresponding target tracking accuracy is also degraded. Furthermore, we can see in Fig. 8 that the total times of radar radiation utilizing the covariance control algorithm is the smallest, but it exhibits the worst target tracking performance. As aforementioned, the proposed

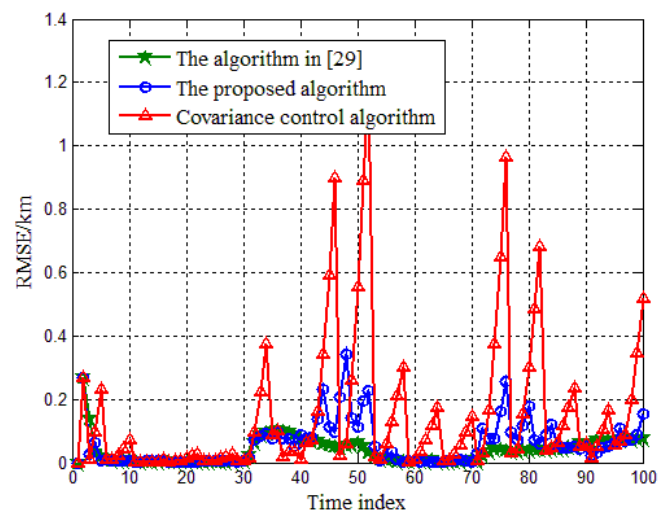

(a)

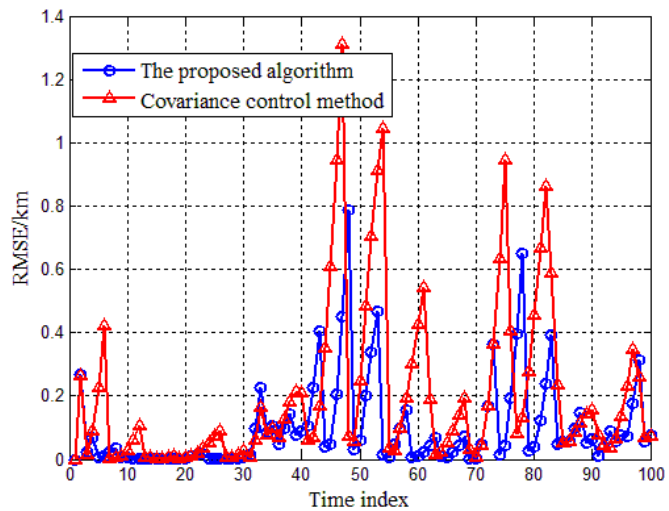

(b)

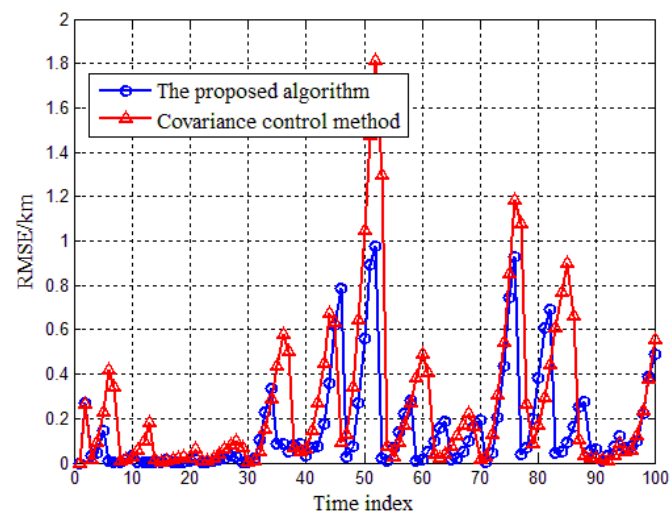

(c)

Fig. 5. Target tracking RMSE in each case. (a) Case 1: $\lambda=1$. (b) Case 2: $\lambda=2$. (c) Case 3: $\lambda=3$.

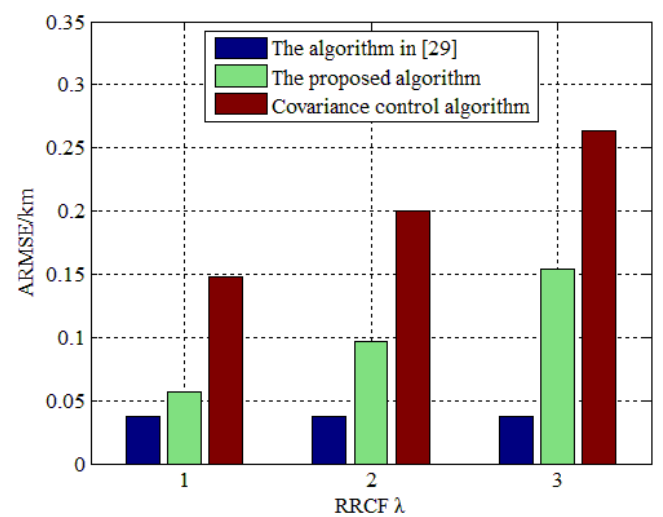

Fig. 6. Target tracking performance of different algorithms. 


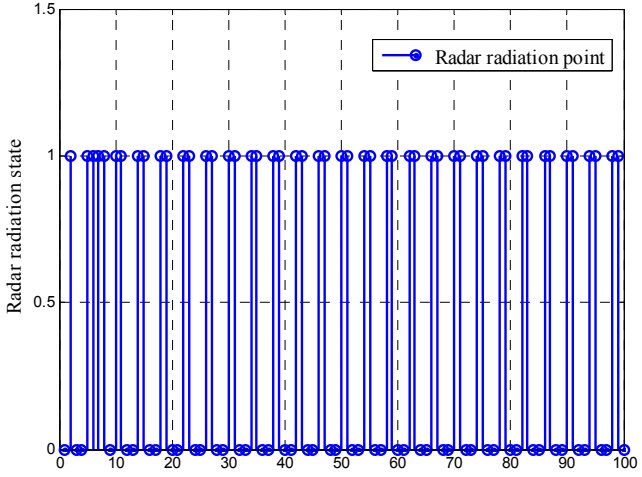

(a)

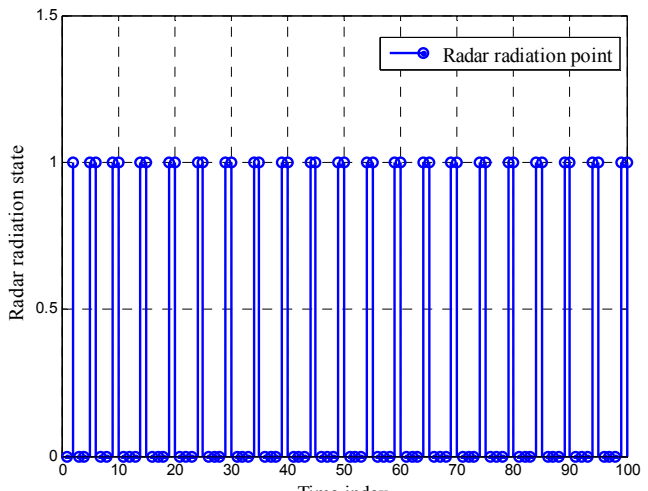

(b)

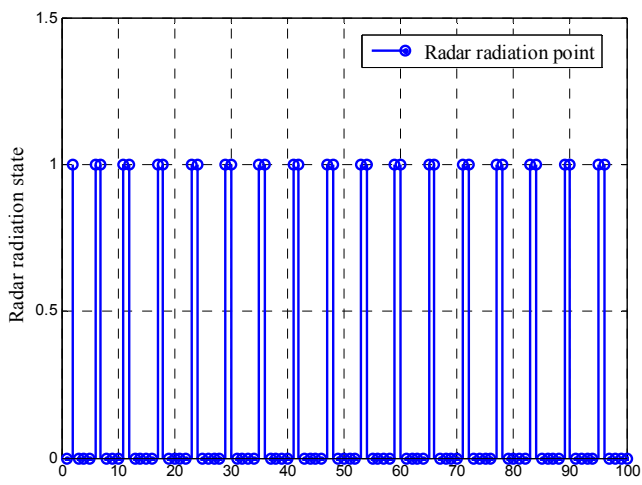

(c)

Fig. 7. Radar real-time radiation state in each case. (a) Case 1: $\lambda=1$. (b) Case 2: $\lambda=2$. (c) Case 3: $\lambda=3$.

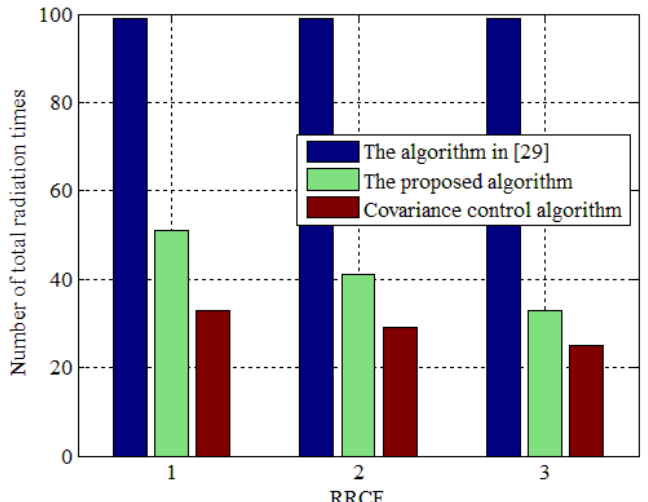

Fig. 8. The number of total radiation times for different algorithms.

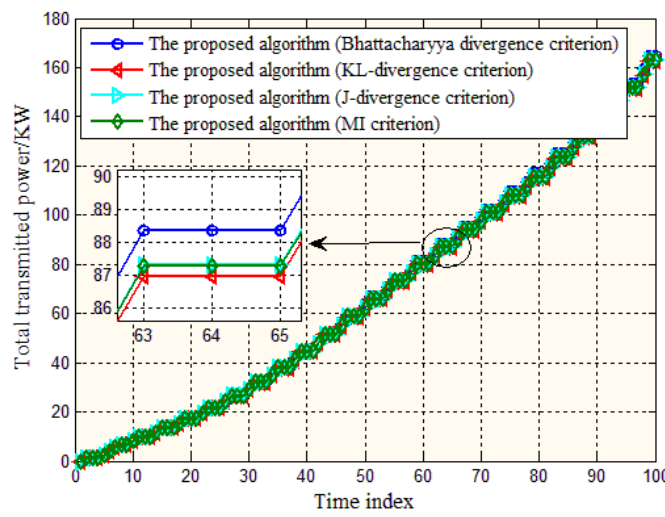

(a)

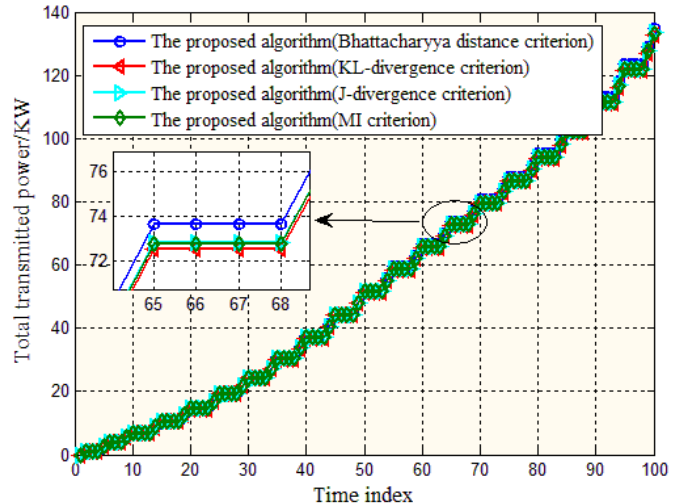

(b)

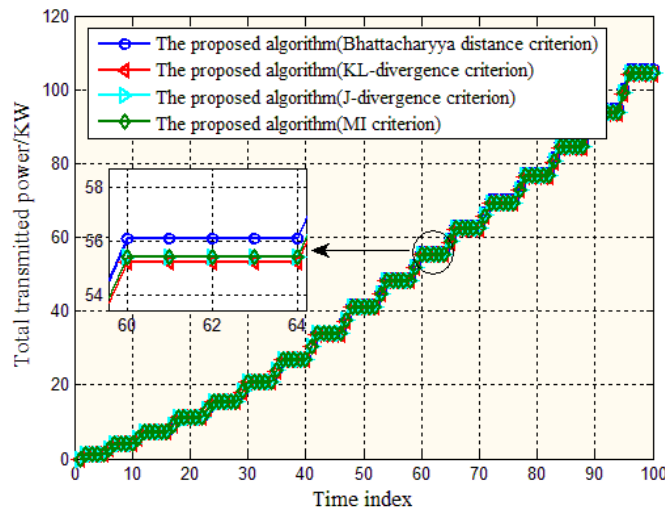

(c)

Fig. 9. The total transmitted power at each time index. (a) Case 1: $\lambda=1$. (b) Case 2: $\lambda=2$. (c) Case 3: $\lambda=3$.

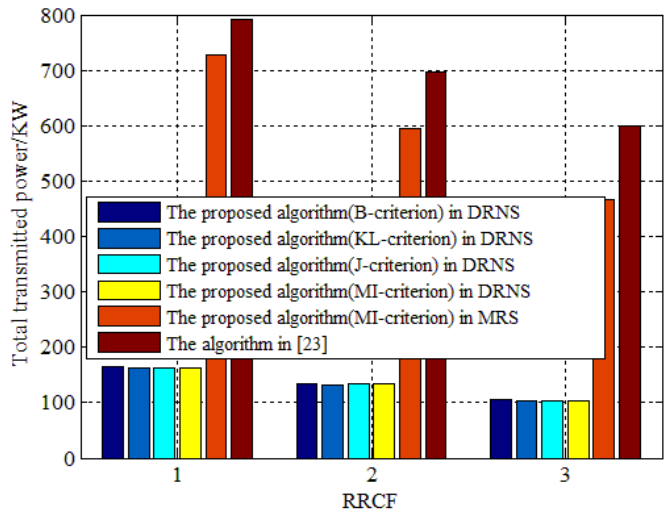

Fig. 10. The total transmitted power of different algorithms. 
algorithm can obtain the best tradeoff between the target tracking accuracy and the total times of radar radiation.

To obtain the optimal transmitted power in DRNS, we employ NPGA to solve the optimization problem [28], where the population size is set to be 100 , the crossover probability is 0.6 , and the mutation probability is 0.01 . The population evolves 10 generations. The parameters are essentially needed to solve the resulting optimization problem by utilizing NPGA. For more details refer to [28].

In order to reveal the effects of RRCF on the total transmitted power results, Figure 9 depicts the total transmitted power results at each time index utilizing our proposed four information-theoretic criteria as RRCF increases. For all the cases, one can see that as RRCF increases, the total transmitted power is reduced. As aforementioned, this is due to the fact that the total times of radar radiation decreases with the increase of the expected error covariance matrix.

Figure 10 shows the total transmitted power of different algorithms. We can see that the total transmitted power utilizing our proposed adaptive resource scheduling algorithm with the information-theoretic criteria in DRNS is significantly smaller than those of other algorithms, which further shows the remarkable LPI improvement by exploiting our presented strategy in DRNS. The proposed algorithm in DRNS transmits only $17 \%-21 \%$ of the total transmitted power by the proposed algorithm in [28]. Besides, the comparison of the total transmitted power between the DRNS and the MRS (the dedicated radar netting station) is also plotted in Fig. 10, where only the MI criterion based adaptive resource scheduling strategy is employed in MRS for brevity. As expected, using the SNR contributions from all transmitter-receiver pairs, the DRNS can provide a better LPI performance.

\section{Conclusions}

In this paper, the problem of adaptive resource scheduling based on TDOA cooperation in DRNS is investigated, which improves the LPI performance in target tracking by optimizing the radar revisit interval and the transmitted power for a predetermined tracking performance. The NPGA is employed to solve the resulting nonconvex and nonlinear optimization problems. Simulation results are provided to demonstrate that, compared with other algorithms, a significant improvement of the LPI performance can be achieved through our proposed strategy. From a practical point of view, in the future, the proposed algorithm can be implemented in satellite communications as well as a system of using reconfigurable antennas and other emerging wireless technologies for finding more accurately targets [46-49]. In addition, the findings in this paper can be implemented in other scientific fields using the tracking technique to find a better cutoff point even using ROC analysis [50]. Future work will also concentrate on the problem of the optimal radar sensor scheduling to improve the LPI performance in DRNS.

\section{Acknowledgments}

The support provided by the Natural Science Fund for Colleges and Universities in Jiangsu Province (Grant No. 15KJB510015), the National Natural Science Foundation of China (Grant No. 61371170, No. 61671239), the Priority Academic Program Development of Jiangsu Higher Education Institutions (PADA) and Key Laboratory of Radar Imaging and Microwave Photonics (Nanjing Univ. Aeronaut. Astronaut.), Ministry of Education, Nanjing University of Aeronautics and Astronautics, Nanjing, 210016, China are gratefully acknowledged.

\section{References}

[1] SHI, C. G., SAlOUS, S., WANG, F., ZHOU, J. J. Cramer-Rao lower bound evaluation for linear frequency modulation based active radar networks operating in a Rice fading environment. Sensors, 2016, vol. 16, no. 12, 2072. DOI: $10.3390 / \mathrm{s} 16122072$

[2] PACE, P. E. Detecting and Classifying Low Probability of Intercept Radar. Boston: Artech House, 2009, p. 342-352.

[3] HAIMOVICH, A. M., BLUM, R. S., CIMINI, L. J. JR. MIMO radar with widely separated antennas. IEEE Signal Processing Magazine, 2008, vol. 25, no. 1, p. 116-129. DOI: 10.1109/MSP.2008.4408448

[4] FISHER, E., HAIMOVICH, A., BLUM, R. S., et al. Spatial diversity in radars-models and detection performance. IEEE Transactions on Signal Processing, 2006, vol. 54, no. 3, p. 823-836. DOI: 10.1109/TSP.2005.862813

[5] NAGHSH, M. M., MODARRES-HASHEMI, M., SHAHBAZ PANAHI, S., SOLTANALIAN, M., STOICA, P. Unified optimization framework for multi-static radar code design using informationtheoretic criteria. IEEE Transactions on Signal Processing, 2013, vol. 61, no. 21, p. 5401-5416. DOI: 10.1109/TSP.2013.2278154

[6] ALMSLMANY, A. E., CAO, Q. S., YANG, C. Y. High speed dim air target detection using airborne radar under clutter and jamming effects. Radioengineering, 2015, vol. 24, no. 2, p. 632-642. DOI: $10.13164 /$ re.2015.0632

[7] LEI, P., HUANG, X. Robust detection of moving human target in foliage-penetration environment based on Hough transform. Radioengineering, 2014, vol. 23, no. 1, p. 3-10.

[8] NIU, R. X., BLUM, R. S., VARSHNEY, P. K., DROZD, A. L. Target localization and tracking in noncoherent multiple-input multiple-output radar systems. IEEE Transactions on Aerospace and Electronic System, 2012, vol. 48, no. 2, p. 1466-1487. DOI: 10.1109/TAES.2012.6178073

[9] MEHMOOD, R., HUDA, N. U., SONG, J., RIAZ, M. M., IQBAL, $\mathrm{N}$. Improved mean shift target localization using true background weighted histogram and geometric centroid adjustment. Radioengineering, 2016, vol. 25, no. 3, p. 612-622. DOI: $10.13164 /$ re. 2016.0612

[10] GODRICH, H., TAJER, A., POOR, H. V. Distributed target tracking in multiple widely separated radar architectures. In 2012 IEEE 7th Sensor Array and Multichannel Signal Processing Workshop (SAM). Hoboken (NJ, USA), 2012, p. 153-156. DOI: 10.1109/SAM.2012.6250453

[11] CHAVALI, P., NEHORAI, A. Scheduling and power allocation in a cognitive radar network for multiple-target tracking. IEEE Transactions on Signal Processing, 2012, vol. 60, no. 2, p. 715 to 728. DOI: 10.1109/TSP.2011.2174989 
[12] YAN, J. K., LIU, H. W., JIU, B., BAO, Z. Power allocation algorithm for target tracking in unmodulated continuous wave radar network. IEEE Sensors Journal, 2015, vol. 15, no. 2, p. 1098-1108. DOI: 10.1109/JSEN.2014.2360039

[13] ZHANG, J., JIN, T., HE, Y., ZHOU, Z. M. A centralized processing framework for foliage-penetration human tracking in multistatic radar. Radioengineering, 2016, vol. 25, no. 1, p. 98-105. DOI: $10.13164 /$ re.2016.0098

[14] CHEN, Y. F., NIJSURE, Y., YUEN, C., CHEW, Y. H., DING, Z. G. Adaptive distributed MIMO radar waveform optimization based on mutual information. IEEE Transactions on Aerospace and Electronic System, 2013, vol. 49, no. 2, p. 1374-1385. DOI: 10.1109/TAES.2013.6494422

[15] NGUYEN, N. H., DOGANCAY, K., DAVIS, L. M. Adaptive waveform selection for multistatic target tracking. IEEE Transactions on Aerospace and Electronic System, 2015, vol. 51, no. 1 , p. 688-700. DOI: 10.1109/TAES.2014.130723

[16] KULPA, J. S. Channel influence mitigation in pseudo-noise waveform design for radar applications. Radioengineering, 2014, vol. 23 , no. 1 , p. 128-133.

[17] GODRICH, H., PETROPulu, A. P., POOR. H. V. Sensor selection in distributed multiple-radar architectures for localization: A knapsack problem formulation. IEEE Transactions on Signal Processing, 2012, vol. 60, no. 1, p. 247-259. DOI: 10.1109/TSP.2011.2170170

[18] RADMARD, M., CHITGARHA, M. M., MAJD, M. N., NAYEBI, M. M. Antenna placement and power allocation optimization in MIMO detection. IEEE Transactions on Aerospace and Electronic System, 2014, vol. 50, no. 2, p. 1468-1478. DOI: 10.1109/TAES.2014.120776

[19] MA, B. T., CHEN, H. W., SUN, B., XIAO, H. T. A joint scheme of antenna selection and power allocation for localization in MIMO radar sensor networks. IEEE Communications Letters, 2014, vol. 18, no. 12, p. 2225-2228. DOI: 10.1109/LCOMM.2014.2365206

[20] SCHLEHER, D. C. LPI radar: fact or fiction. IEEE Aerospace and Electronic Systems Magazine, 2006, vol. 21, no. 5, p. 3-6. DOI: 10.1109/MAES.2006.1635166

[21] LYNCH, D. JR. Introduction to RF stealth. Sci Tech Publishing, 2004, p. 20-78. DOI: $10.1049 /$ SBRA120E

[22] STOVE, A. G., HUME, A. L., BAKER, C. J. Low probability of intercept radar strategies. IEE Proceedings of Radar, Sonar and Navigation, 2004, vol. 151 , no. 5, p. 249-260. DOI: 10.1049/iprsn:20041056

[23] LAWRENCE, D. E. Low probability of intercept antenna array beamforming. IEEE Transactions on Antennas and Propagation, 2010, vol. 58, no. 9, p. 2858-2865. DOI: 10.1109/TAP.2010.2052573

[24] ZHANG, Z. K., ZHOU, J. J. A novel LPI method of radar's energy control. Progress in Electromagnetics Research C, 2012, vol. 33, p. 81-94. DOI:10.2528/PIERC12080303

[25] ZHANG, Z. K., ZHU, J. H., TIAN, Y. B., LI, H. L. Novel sensor selection strategy for LPI based on an improved IMMPF tracking method. Journal of Systems Engineering and Electronics, 2014 vol. 25, no. 6, p. 1004-1010. DOI: 10.1109/JSEE.2014.00115

[26] CHEN, J., WANG, F., ZHOU, J. J., SHI, C. G. A novel radar radiation control strategy based on passive tracking in multiple aircraft platforms. In 2014 IEEE China Summit \& International Conference on Signal and Information Processing (ChinaSIP). Xian (China), 2014, p. 777-780. DOI: 10.1109/ChinaSIP.2014.6889350

[27] WU, W., WANG, G. H., LIU, Y., LI, S. Z. Airborne radar/IRST/ESM synergistic tracking and management. Systems
Engineering and Electronics, 2011, vol. 33, no. 7, p. 1517-1522. DOI: 10.3969 / j.issn.1001-506X.2011.07.16 (in Chinese)

[28] SHI, C. G., WANG, F., SELlATHURAI, M., ZHOU, J. J. LPI optimization framework for target tracking in radar network architectures using information-theoretic criteria. International Journal of Antennas and Propagation, 2014, 10 p. DOI: $10.1155 / 2014 / 654561$

[29] SHI, C. G., ZHOU, J. J., WANG, F. Low probability of intercept optimization for radar network based on mutual information. In 2014 IEEE China Summit \& International Conference on Signal and Information Processing (ChinaSIP), 2014, p. 683-687. DOI: 10.1109/ChinaSIP.2014.6889331

[30] SHI, C. G., WANG, F., ZHOU, J. J., ZHANG, H. Security information factor based low probability of identification in distributed multiple-radar system. In IEEE International Conference on Acoustics, Speech and Signal Processing. Brisbane (Australia), 2015, p. 3716-3720. DOI: 10.1109/ICASSP.2015.7178665

[31] NARYKOV, A. S., KRASNOV, O. A., YAROVOY, A. Algorithm for resource management of multiple phased array radars for target tracking. In The 16th International Conference on Information Fusion. Istanbul (Turkey), 2013, p. 1258-1264.

[32] NARYKOV, A. S., YAROVOY, A. Sensor selection algorithm for optimal management of the tracking capability in multisensor radar system. In Proceedings of the 43rd European Microwave Conference. Nuremberg (Germany), 2013, p. 1811-1814. DOI: 10.23919/EuMC.2013.6687031

[33] ZHANG, Z. N., SHAN, G. L. Non-myopic sensor scheduling to track multiple reactive targets. IET Signal Processing, 2015, vol. 9, no. 1, p. 37-47. DOI: 10.1049/iet-spr.2013.0187

[34] FAWCETT, T. An introduction to ROC analysis. Pattern Recognition Letters, 2006, vol. 27, no. 8, p. 861-874. DOI: 10.1016/j.patrec.2005.10.010

[35] HAND, D. J., TILL, R. J. A simple generalisation of the area under the ROC curve for multiple class classification problems. Machine Learning, 2001, vol. 45, no. 2, p. 171-186. DOI: 10.1023/A:1010920819831

[36] WU, P. L., GUO, Q., ZHANG, X. Y., BO, Y. M. Maneuvering target tracking using passive TDOA measurements. In Proceedings of the 33rd Chinese Control Conference. Nanjing (China), 2014, p. 758-762. DOI: 10.1109/ChiCC.2014.6896722

[37] SHI, C. G., ZHOU, J. J., WANG, F. LPI based resource management for target tracking in distributed radar network. In IEEE Radar Conference (RadarConf). Philadelphia (PA, USA), 2016, p. 822-826. DOI: 10.1109/RADAR.2016.7485222

[38] TOBES, Z., RAIDA, Z. Use of the analog neural networks in the adaptive antenna control systems. Radioengineering, 2002, vol. 11, no. 3, p. 14-21.

[39] TOBES, Z., RAIDA, Z. Improvements of analog neural networks based on Kalman filter analysis. Radioengineering, 2002, vol. 11, no. 1 , p. 6-13.

[40] BISWAS, S. K., QIAO, L., DEMPSTER, A. G. A novel a priori state computation strategy for the unscented Kalman filter to improve computational efficiency. IEEE Transactions on Automatic Control, 2017, vol. 62, no. 4, p. 1852-1864. DOI: 10.1109/TAC.2016.2599291

[41] GUO, Z., DONG, C. Y., CAI, Y. L., YU, Z. H. Time-varying transition probability based IMM-SRCKF algorithm for maneuvering target tracking. Journal of Systems Engineering and Electronics, 2015, vol. 37, no. 1, p. 24-30. DOI: 10.3969/j.issn.1001-506X.2015.01.05 (In Chinese)

[42] CHITGARHA, M. M., RADMARD, M., MAJD, M. N., NAYEBI, M. M. Improving MIMO radar's performance through receivers' 
positioning. IET Signal Processing, 2017, vol. 11, no. 5, p. 622 to 630. DOI: 10.1049 /iet-spr.2016.0556

[43] WANG, X., HAN, C. Z. A VS-MIMO algorithm for maneuvering target tracking. In The 2nd International Conference on Industrial Mechatronics and Automation. Wuhan (China), 2010, vol. 1, p. 334-337.

[44] SONG, X. F., WILlETT, P., ZHOU, S. L. Optimal power allocation for MIMO radars with heterogeneous propagation losses. In IEEE International Conference on Acoustics, Speech and Signal Processing. Kyoto (Japan), 2012, p. 2465-2468. DOI: 10.1109/ICASSP.2012.6288415

[45] SONG, X. F., WILLETT, P., ZHOU, S. L., GLAZ, J. MIMO radar detection with heterogeneous propagation losses. In IEEE Statistical Signal Processing Workshop. Ann Arbor (MI, USA), 2012, p. 776-779. DOI: 10.1109/SSP.2012.6319819

[46] RAPTIS, V., VOTIS, C., TATSIS, G., CHRONOPOULOS, S. K., CHRISTOFILAKIS, V., KOSTARAKIS, P. Active tuning antennas for wireless communication. In AIP Conference Proceedings, 2010, vol. 1203, no. 1, p. 1058-1062. DOI: $10.1063 / 1.3322310$

[47] RAPTIS, V., TATSIS, G., VOTIS, C., CHRONOPOULOS, S. K., CHRISTOFILAKIS, V., KOSTARAKIS, P. Tuning techniques for planar antennas in wireless communication. In AIP Conference Proceedings, 2010, vol. 1203, no. 1, p. 1053-1057. DOI: $10.1063 / 1.3322309$

[48] RAPTIS, V., TATSIS, G., CHRONOPOULOS, S. K., MALLIOS, S., KOSTARAKIS, P. Development and experimental measurements of a tunable antenna. Communications and Network, 2013, vol. 5, no. 3, p. 220-224. DOI: $10.4236 / \mathrm{cn} .2013 .53026$
[49] BANERJEE, S., SHARIF, H. Communication subsystems for emerging wireless technologies. Radioengineering, 2012, vol. 21, no. 4, p. 1036-1049.

[50] TAFIADIS, D., CHRONOPOULOS, S.K., KOSMA, E. I., et al. Using receiver operating characteristics curve to define the cutoff points of voice handicap index applied to young adult male smokers. Journal of Voice, 2017, DOI: 10.1016/j.jvoice.2017.06.007

\section{About the Authors ...}

Jing TAN was born in 1978. She received her B.S. from Nanjing University of Aeronautics and Astronautics (NUAA) in 2005, and she is currently working toward her $\mathrm{Ph} . \mathrm{D}$. degree in NUAA. Her main research interest is antenna array, aircraft radio frequency stealth, radar target tracking.

Chenguang SHI was born in 1989. He received his M.S. and Ph.D. from NUAA in 2012 and 2017 respectively, and then became a lecturer there. His main research interest is aircraft radio frequency stealth, radar signal processing, sensor networks, and information fusion.

Jianjiang ZHOU was born in 1962. He received his M.S. and Ph.D. from NUAA in 1988 and 2001 respectively, and then became a professor there. His main research interest is aircraft radio frequency stealth, radar signal processing. 